\title{
The ten reasons why corticosteroid therapy reduces mortality in severe COVID-19. Author's reply
}

\author{
G. Umberto Meduri ${ }^{1,2^{*}}$, George P. Chrousos ${ }^{3}$ and Yaseen M. Arabi ${ }^{4}$ (D)
}

(0) 2021 European Society of Intensive Care Medicine (ESICM), European Society for Pediatric and Neonatal Intensive Care (ESPNIC) and Springer-Verlag GmbH Germany, part of Springer Nature

The letter by Añón and Villar [1] raises important issues that were outside the scope of our Commentary. A recent publication in this journal on the pharmacological principles guiding prolonged glucocorticoid (GC) treatment in acute respiratory distress syndrome (ARDS) addresses these matters [2]. GCs are agonist compounds that bind to the $\mathrm{GC}$ receptor (GR), producing a pharmacological response. Clinical efficacy depends on the magnitude and duration of exposure of the GR. The review addresses the roles that dosage, timing of initiation, mode of administration, duration, and dose tapering, may play in achieving optimal response to GC therapy in ARDS [2]. Based on randomised controlled trials' (RCTs) findings, GC plasma concentration-time profiles, and pharmacodynamic studies, optimal results are most likely achievable with early intervention and a dose sufficient to achieve close to maximal GR saturation (methylprednisolone 80-100 mg, dexamethasone $20 \mathrm{mg}$ ) [2]. Therefore, dexamethasone at a dose similar to the landmark DEXAARDS RCT [3] might achieve even better outcomes than the one reported in the RECOVERY RCT [4]. The evidence does not support the concern about complications from higher doses (methylprednisolone equivalent 80 -to- $160 \mathrm{mg}$ ) or prolonged (28 days) treatment [2]. On the contrary, ARDS RCTs investigating higher doses and longer duration of treatment reported a lower rate of complications, in part explained by the shorter duration of ventilatory dependence [2]. In coronavirus disease

\footnotetext{
${ }^{*}$ Correspondence: umeduri@utmem.edu

${ }^{1}$ Memphis Veterans Affairs Medical Center Research Service

and Pulmonary, Critical Care, and Sleep Medicine Service, Memphis, TN, USA

Full author information is available at the end of the article
}

2019 (COVID-19), extended GC treatment was not associated with impaired viral clearance [5].

We agree with the authors that morbidity-mortality follow-up data beyond 28 days are important in a disease associated with prolonged mechanical ventilation (MV) and long-term disability. It is also likely that discontinuing GC treatment on day ten, while still on ventilatory support, lessens treatment's potential benefits. We believe that $\mathrm{GC}$ treatment directed at improving longterm outcomes should take into consideration the recent discoveries underscoring the central regulatory function of GC-activated GR in critical illness throughout disease development and resolution [6]. Based on these findings, GC treatment should probably be prolonged to support disease resolution (restoration of tissue anatomy/structure and function), an active process associated with multiple biochemical pathways, including switching production from pro-inflammatory to pro-resolving mediators with the resolution of granulation tissue, restoration of tissue integrity, and clearance of apoptotic cells and debris.

In a recent longitudinal observational study [5] conducted in 14 Italian Respiratory Units $(n=173)$, a protocol with prolonged methylprednisolone at a dose of $80 \mathrm{mg} /$ day, administered in patients with severe COVID19 pneumonia and high levels of systemic inflammation, demonstrated a $71 \%$ reduction in mortality and increased ventilation-free days by study day 28 [5]. The treatment was well tolerated and did not affect viral clearance from the airways.

The MEDEAS trial (Methylprednisolone vs. Dexamethasone in COVID-19 Pneumonia trial, ClinicalTrials.gov Identifier: NCT04636671) attempts to address this issue by comparing the RECOVERY RCT protocol

\section{Springer}


to a protocol similar to the one investigated in the Italian prospective observational study [5]. The study drug is methylprednisolone given as an initial bolus of $80 \mathrm{mg}$ to achieve close-to-maximal GR $\alpha$ saturation, followed by a continuous infusion to maintain high response levels throughout treatment, with the option of adjusting treatment duration based on parameters of clinical severity and followed by dosage gradual de-escalation to avoid inflammation rebound. Site recruitment is still in progress. Completion of this RCT should provide clarity on how essential components of the GC treatment protocol impact short and long-term outcomes.

\section{Author details}

${ }^{1}$ Memphis Veterans Affairs Medical Center Research Service and Pulmonary, Critical Care, and Sleep Medicine Service, Memphis, TN, USA. ${ }^{2}$ Department of Medicine, University of Tennessee Health Science Center, Memphis, TN, USA. ${ }^{3}$ University Research Institute of Maternal and Child Health and Precision Medicine, Medical School, National and Kapodistrian University of Athens, Athens, Greece. ${ }^{4}$ Intensive Care Department, Ministry of National Guard Health Affairs, King Abdullah International Medical Research Center, King Saud Bin Abdulaziz University for Health Sciences, Riyadh, Kingdom of Saudi Arabia.

\section{Compliance with ethical standards}

\section{Conflicts of interest}

The authors have no financial or personal relationships with other people or organizations that could inappropriately influence (bias) their work.

\section{Publisher's Note}

Springer Nature remains neutral with regard to jurisdictional claims in published maps and institutional affiliations.

Accepted: 9 December 2020

Published online: 28 January 2021

\section{References}

1. Anon JM, Villar J. Ten reasons why corticosteroid therapyreduces mortality in severe COVID-19. Intensive Care Med. https://doi.org/10.1007/s0013 4-020-06330-w

2. Meduri GU, Annane D, Confalonieri M, Chrousos GP, Rochwerg B, Busby A, Ruaro B, Meibohm B (2020) Pharmacological principles guiding prolonged glucocorticoid treatment in ARDS. Intensive Care Med 46:2284-2296

3. Villar J, Ferrando C, Martinez D, Ambros A, MunozT, Soler JA, Aguilar G, Alba F, Gonzalez-Higueras E, Conesa LA et al (2020) Dexamethasone treatment for the acute respiratory distress syndrome: a multicentre, randomised controlled trial. Lancet Respirat Med 8(3):267-276

4. Horby P, Lim WS, Emberson J, Mafham M, Bell J, Linsell L, Staplin N, Brightling C, Ustianowski A, Elmahi E (2020) Dexamethasone in hospitalized patients with Covid-19-preliminary report. N Engl J Med. https://doi. org/10.1056/NEJMoa2021436

5. Salton F, Confalonieri P, Meduri GU, Santus P, Harari S, Scala R, Lanini S, Vertui V, Oggionni T, Caminati A (2020) Prolonged low-dose methylprednisolone in patients with severe COVID-19 pneumonia. In: open forum infectious diseases: Oxford University press US, vol 7, ofaa421

6. Meduri GU, Chrousos GP (2020) General adaptation in critical illness: glucocorticoid receptor-alpha master regulator of homeostatic corrections. Front Endocrinol (Lausanne) 11:161 